\title{
A importância das plantas medicinais para a comunidade pesqueira de Algodoal ${ }^{1}$
} The importance of medicinal plants for the Algodoal fishing community

\author{
André Luís Cote Roman ${ }^{\mathrm{I}}$ \\ João Ubiratan Moreira dos Santos II
}

Resumo: Nas últimas três décadas, a população de pescadores artesanais da Zona do Salgado, litoral paraense, tem passado por um processo de mudança cultural, social e econômica, embora tenha persistido o uso de plantas para diversos fins. Os objetivos deste estudo foram indicar as espécies de restinga mais utilizadas para fins terapêuticos pelos moradores de Algodoal, comunidade pesqueira inserida na área geográfica em questão e testar técnicas etnobotânicas combinadas para esta finalidade. Através desta pesquisa foram registradas 24 espécies com valor medicinal, das quais 8 destacaram-se no que tange à importância cultural.

Palavras-chave: Restinga. Amazônia. Comunidade pesqueira. Plantas medicinais. Etnobotânica.

Abstract: Over the past three decades the fishermen of the Salgado, region on the Pará coast, have experienced a process of cultural, social and economic change, although the use of plants for various purposes has persisted. The aims of this study were to identify the species of restinga which are most widely used for medicinal purposes by the residents of Algodoal, a fishing community located in the region in question, and to test ethnobotanical techiniques designed for this objective. During the study, 24 species with medicinal properties were identified, of which eight were outstanding in terms of cultural importance.

Keywords: Restinga. Amazônia. Fishing community. Medicinal plants. Ethnobotany.

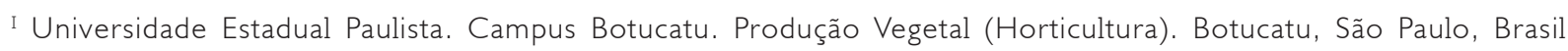
(coteroman@yahoo.com.br).

II Museu Paraense Emílio Goeldi. Coordenação de Botânica. Pesquisador. Belém, Pará, Brasil (bira@museu-goeldi.br).

1 Parte da dissertação de mestrado do primeiro autor, do curso de pós-graduação da Universidade Federal Rural da Amazônia (UFRA).
}

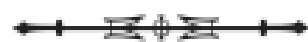




\section{INTRODUÇÃO}

A adaptação humana ao litoral brasileiro parece ter sido um dos modos de vida mais bem sucedidos e estáveis, pois estendeu-se por mais de nove mil anos e, mais do que a caça e a coleta nos campos, cerrados e matas do interior, garantiu a sobrevivência de uma população numerosa (FAJARDO, 2002).

A importância do reino vegetal para os povos que habitaram a costa brasileira, desde tempos mais remotos (coletores de moluscos e pescadores), é verificada através de testemunhos arqueológicos, como os sambaquis, do tupi 'depósitos de conchas' (KNEIP, 1987; SCHIMTZ, 1989). Há indícios de que esses grupos realizavam manejo de plantas e, provavelmente, protegiam algumas espécies apreciadas para consumo ou fabricação de artefatos (GASPAR, 2000).

Com a chegada dos colonizadores, a exploração dos recursos naturais ampliou-se e, cada vez mais, os complexos e frágeis ecossistemas litorâneos foram sendo degradados. Hoje, o potencial de uso das espécies vegetais das restingas para alimentação, fabricação de produtos agrícolas, produção de fármacos, resinas e corantes, entre outros é superficialmente conhecido (ARAÚJO; LACERDA, 1987; GOTLLIEB; KAPLAN, 1990).

A etnobotânica, disciplina encarregada de pesquisar a interação ser humano-planta (FORD, 1978), tem se ocupado com o resgate desses conhecimentos, os quais foram acumulados e transmitidos por gerações e que hoje passam por um processo de desaparecimento acelerado (XOLOCOTZY, 1983). Assim, tem também contribuído, entre outras coisas, no auxílio a pesquisadores da área de ecologia, em trabalhos de conservação ambiental (GADGIL; BERKES; FOLKE, 1993; COHN, 1988; HALFFTER, 1985) e na seleção de espécies medicinais para pesquisa, envolvendo etapas lentas e dispendiosas, inerentes a estes estudos (ALEXIADES, 1996).
No Brasil, trabalhos etnobotânicos realizados em zonas litorâneas são raros, principalmente quando se considera a riqueza florística de seus ecossistemas (ARAÚJO; LACERDA, 1987). Junto a isto, deve-se ressaltar que as populações de pescadores artesanais, habitantes destas regiões, guardam, muitas vezes, heranças de conhecimentos e procedimentos relativos ao uso de plantas provenientes de grupos indígenas há muito extintos e que hoje estão ameaçadas pela alteração do modo de vida tradicional de seus habitantes (PRANCE, 1991).

Fonseca e Sá (1997), inventariando os trabalhos de etnobotânica e botânica econômica existentes em áreas costeiras no Brasil, constataram que $80 \%$ dos estudos realizados em restingas encontram-se na porção sudeste, uma pequena faixa do litoral brasileiro, evidenciando, assim, a enorme carência dessas investigações em outras regiões do país. Segundo os autores, esta situação agrava-se quando se observam questões metodológicas, verificando que, não raro, muitas pesquisas apresentam uma relação pontual de plantas úteis, não se ocupando com a identificação social e aspectos culturais, muitas vezes específicos de cada local e que são, conforme Fleming-Moran (1992), essenciais para o uso coerente das plantas.

Na Amazônia, mais precisamente no estado do Pará, a intensificação da atividade turística em locais como Marudá, Algodoal, Salinas e Ajuruteua, que se inserem na chamada Zona Fisiográfica do Salgado, revela, também, uma necessidade urgente de estudos desta natureza (BASTOS; ROSÁRIO; LOBATO, 1995). Nestas áreas, os trabalhos realizados em restinga têm focalizado, sobretudo, aspectos florísticos e fitossociológicos (BRAGA, 1979; SANTOS; ROSÁRTO, 1988; BASTOS, 1988; COSTA NETO; BASTOS; LOBATO, 1996; BASTOS; ROSÁRIO; LOBATO, 1995; BASTOS, 1996; AMARAL, 1997; COSTA NETO, 1999; ROCHA, 2000; AMARAL, et al., 2001 a, AMARAL et al., 2001 b; COSTA NETO et al., 2001) sem dar atenção, no entanto, ao potencial de utilização das espécies estudadas. Contudo, alguns

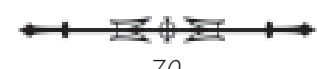


trabalhos etnobotânicos envolvendo ecossistemas de restingas já foram realizados na região (FURTADO; SOUZA; BERG, 1978; BASTOS, 1995; SANTOS et al., 1997; URQUIZA et al., 1999; FUNTEC/ SECTAM, 2000; COELHO-FERREIRA, 2000), sendo o de Bastos (1995) o único a tratar, exclusivamente, das espécies nativas.

Essa carência de estudos explica uma necessidade cada vez maior da intensificação das pesquisas etnobotânicas nestes ambientes. O presente trabalho concentrou-se na vila de pescadores de Algodoal e teve como objetivos determinar a importância das espécies medicinais de uma área de restinga na cultura local e testar técnicas etnobotânicas combinadas para essa finalidade.

\section{MATERIAL E MÉTODOS}

\section{Caracterização ambiental da área de estudo}

A ilha de Algodoal, município de Maracanã, está situada na Zona Fisográfica do Salgado, porção litorânea do Pará, a leste da desembocadura do rio Amazonas (SIMÕES, 1981; FURTADO, 1987). A excelente vocação à pesca que essa posição geográfica possui explica a existência na ilha de uma população de aproximadamente 1.600 habitantes, distribuídas em quatro comunidades: Vila de Algodoal, com cerca de 1.000 habitantes; Fortalezinha, Mocoóca e Camboinha, juntas, com aproximadamente 600 habitantes (VIANNA; MORGADO, 2001).

A ilha de Algodoal-Maiandeua, com uma área total de 2.378 ha, foi declarada Área de Proteção Ambiental (APA), unidade de conservação de caráter insular, sob a Lei estadual $n^{\circ}$ 5621, de 25 de novembro de 1990 (BASTOS, 1996).

A área onde se concentrou a pesquisa está localizada na porção norte da itha, na denominada 'Praia da Princesa' (Figura 1), local intimamente ligado ao cotidiano dos moradores de Algodoal. A delimitação da restinga estudada fez-se da zona de preamar até

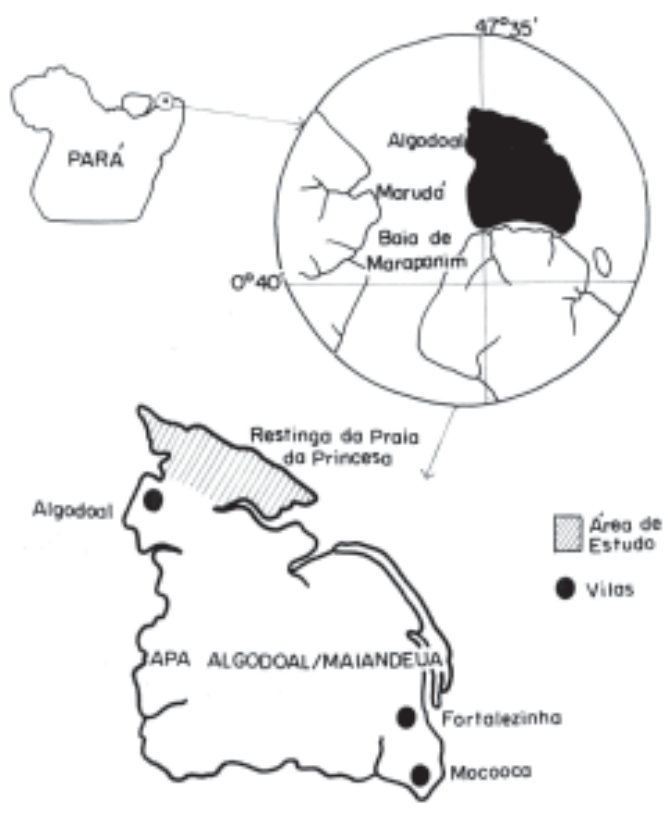

ESCALA: I: 100000

Figura 1. Localização da área de estudo: praia da Princesa, ilha de Algodoal, município de Maracanã, Pará. Fonte: Rocha (2000).

o contato com o manguezal, percorrendo cerca de $800 \mathrm{~m}$ e abrangendo as seis formações vegetais, descritas por Bastos (1996): psamófila reptante, brejo herbáceo, formação de dunas, campo entre dunas, campo arbustivo aberto e mata de restinga.

\section{Aspectos gerais e históricos}

Os moradores de Algodoal e de outras localidades da Zona do Salgado apresentam-se com uma organização social fortemente dependente dos recursos marinhos (FURTADO, 1987), tendo como principal fonte econômica a pesca de subsistência. A ausência de tecnologia moderna empregada nesta atividade fazem com que sejam conhecidos como pescadores artesanais (MALDONADO, 1995), embora os habitantes autodenominem-se apenas de pescadores.

Ainda que não haja registros de descoberta arqueológica, acredita-se que a itha de Algodoal tenha

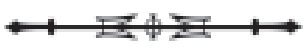


sido, inicialmente, ocupada por Tupinambás do grupo dos Pacajás, pois algumas localidades próximas, como Marapanim, Maracanã e Marudá, foram estabelecidas a partir desses povos indígenas (FURTADO, 1981).

Desta forma, ainda predominam no Salgado aspectos culturais de uma colonização que se deu através do contato interétnico, sobretudo entre europeus e povos indígenas (CORRÊA, et al., 1994; ALVES FILHO; ALVES JUNIOR; MAIA NETO, 1999) e que hoje representam, conforme Parker (1989), um importante sistema adaptativo ao meio natural pertinente.

O turismo predatório, no entanto, intensificado nesta área geográfica a partir do final da década de 1980, tem promovido, além da degradação ambiental, alterações nos costumes e tradições da população nativa. Em Algodoal, situada a cerca de $200 \mathrm{~km}$ de Belém, essas mudanças são reveladas, entre outros, pelo abandono dos padrões tradicionais de construção de suas habitações e, em alguns casos, da própria atividade pesqueira, substituída por ocupações ligadas ao turismo.

\section{Técnicas etnobotânicas}

Para determinar as espécies medicinais da restinga da Princesa, utilizadas pela população de Algodoal, foi realizado um inventário etnobotânico auxiliado por dois colaboradores locais, reconhecidos pela população como os detentores de maior experiência e tradição no uso da flora nativa. As técnicas de coleta seguiram as descritas em Martin (1995) e Ming (1996). Para o tratamento taxonômico foi utilizado o sistema de classificação de Cronquist (1981), com exceção das Leguminosae, que seguiu a classificação de Engler e Prantl (1894). O material botânico foi incorporado ao Herbário João Murça Pires, da Coordenação de Botânica do Museu Paraense Emílio Goeldi (MPEG), e ao Herbário da Universidade Federal Rural da Amazônia (UFRA).

A importância cultural das espécies da restinga foi verificada através da combinação de duas técnicas etnobotânicas: entrevista semi-estruturada (free listing), descrita por Alexiades (1996), e entrevista estruturada (BERNARD, 1988 apud ALEXIADES, 1996). Os questionários foram aplicados a 30 casais residentes nas três principais ruas da vila.

A técnica do free listing teve como finalidade posicionar o uso das plantas de restinga dentro do sistema terapêutico como um todo, considerando que as populações tradicionais, de acordo com Amorozo e Gèly (1988), exploram várias fontes de espécies com valor medicinal (Apêndice 1). A identificação das espécies não ocorrentes na restinga, geralmente cultivadas em quintais, foi realizada utilizando os nomes populares citados pelos entrevistados e comparando-os com os citados no levantamento da Funtec/Sectam (2000), o qual aborda as plantas medicinais de Algodoal de modo geral, sem focalizar um ecossistema específico, finalidade essencial do presente trabalho.

Os questionários estruturados, contendo apenas os nomes das espécies inventariadas na restinga da Princesa (Apêndice 2), foram aplicados aos mesmos entrevistados para verificar se eram conhecidas e utilizadas. Neste caso, para o reconhecimento das espécies pelos informantes, foram apresentadas, durante as entrevistas, amostras das plantas através de um 'herbário móvel' e fotografias.

\section{RESULTADOS E DISCUSSÃO}

\section{Inventário etnobotânico}

Através do inventário etnobotânco foram identificadas na restinga da Princesa 24 espécies medicinais, distribuídas em 19 famílias (Apêndice 3). A população de Algodoal utiliza, contudo, plantas vindas de outros ambientes, totalizando, segundo os dados recolhidos pela técnica do free listing, 80 espécies para fins terapêuticos (Apêndice 4).

Na Tabela 1 são apresentadas as dez mais citadas, dentre as quais, três ocorrem na Restinga da Princesa e sete constituem-se de plantas exóticas, comumente cultivadas em quintais.

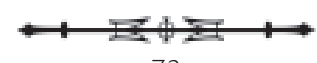


Tabela 1. Espécies medicinais mais citadas pela população de Algodoal, município de Maracanã, Pará.

\begin{tabular}{|c|c|c|}
\hline Nome específico & $\begin{array}{l}\text { Nome } \\
\text { popular }\end{array}$ & $\begin{array}{l}\text { \% dos } \\
\text { entrevistados } \\
\text { que as citaram }\end{array}$ \\
\hline Ocimum minimum L. & Manjericão & 32 \\
\hline $\begin{array}{l}\text { Himatanthus articulata (Vahl.) } \\
\text { Woodson }\end{array}$ & Sucuba * & 30 \\
\hline Ocimum cf. selloi Benth & Alfavacão & 22 \\
\hline Mentha pulegium L. & Hortelãzinho & 22 \\
\hline Lippia alba (Mill.) N.E.Br. & Erva-cidreira & 20 \\
\hline Anacardium occidentale L. & Caju * & 20 \\
\hline Chenopodium ambrosioides $\mathrm{L}$. & Mastruz & 18 \\
\hline Plectranthus barbatus Benth. & Anador & 18 \\
\hline $\begin{array}{l}\text { Dalbergia ecastophyllum (L.) } \\
\text { Taub. }\end{array}$ & Verônica * & 17 \\
\hline Arrabidaea chica Verl. & Pariri & 15 \\
\hline
\end{tabular}

* espécie ocorrente na restinga da Princesa

O número de plantas introduzidas representa cerca de $85 \%$ de todas as espécies citadas (Apêndice 4), com resultados próximos aos encontrados por Furtado, Souza e Berg (1978), Santos et al. (1997), Urquiza et al. (1999), Funtec/Sectam (2000) e Coelho-Ferreira (2000), todos na Zona do Salgado. Isso indica uma flexibilidade cultural refletida pela grande interação desta microrregião com outras comunidades e o forte intercâmbio de plantas ocorrido, possivelmente, por influência européia durante o processo de colonização.

Ao comparar esses dados com os encontrados na costa sudeste da Mata Atlântica (Rio de Janeiro), por Begossi, Leitão Filho e Richerson (1993), Begossi (1998a), Figueiredo et al. (1993) e Figueiredo, Leitão Filho e Richerson (1997), verificou-se que os resultados são semelhantes, comprovando que grupos originados de duas ou mais tradições culturais, como é o caso dos pescadores artesanais da costa brasileira, apresentam grande riqueza cultural em relação ao uso de plantas, indicando uma maior gama de opções adaptativas.

No entanto, uma maior ou menor dependência dos recursos naturais locais pode, conforme Begossi (1998b), influenciar o conhecimento contemporâneo das populações tradicionais. Com relação a esse aspecto, segue-se a discussão com o foco sobre o uso espeć́fico das espécies ocorrentes na restinga da Princesa.

\section{Plantas da restinga da Princesa}

A importância cultural das espécies da restinga da Princesa, quando investigada pela técnica do free listing, evidencia-se de modo diferente em relação à técnica de entrevista estruturada. Para o primeiro caso, 11 das espécies da restinga (Figura 2), o que corresponde a $46 \%$ das inventariadas nesse ecossistema, apareceram na lista das 80 citadas pelos entrevistados. É importante ressaltar que essa investigação deu-se utilizando uma questão semi-aberta ("Quais as dez plantas que você mais usa para fim medicinal?"), sem nenhum outro tipo de especificação. Deste modo, ampliaram-se as opções de resposta por parte do entrevistado com relação ao número de espécies que utilizam em seu cotidiano.

Por outro lado, quando a investigação fez-se de forma direcionada, trabalhando unicamente com as 24 espécies estudadas, a importância cultural de

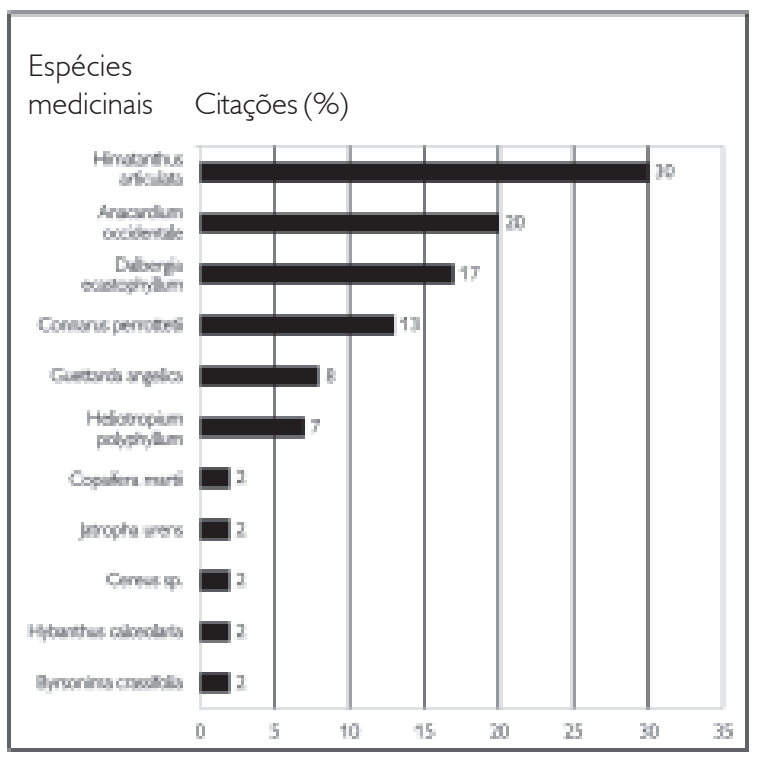

Figura 2. Espécies medicinais levantadas na restinga da Princesa, Algodoal, município de Maracanã, Pará e a porcentagem dos entrevistados (60) que as citaram. Resultado do free-listing.

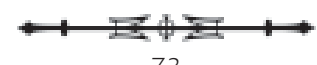


algumas espécies apresentou-se mais evidente. A primeira diferença é que, neste caso, todas as espécies foram, pelo menos uma vez, citadas pelos entrevistados (Figura 3).

No que se refere às espécies mais citadas, a utilização do free listing foi importante, pois conseguiu ressaltar a importância cultural de três espécies ocorrentes na restinga para a população de Algodoal. Isto significa que, apesar de investigadas dentro de um amplo universo de plantas consideradas, a sucuba (Himatanthus articulata), o caju (Anacardium occidentale) e a verônica (Dalbergia ecastophyllum) apresentaram-se entre as dez mais citadas pela população, sendo esse resultado confirmado quando investigadas de forma direcionada.

No entanto, algumas espécies apresentaram-se com forte importância cultural somente quando foram investigadas de forma direta, como é o caso da sete sangrias (Heliotropium polyphyllum), do pocoró

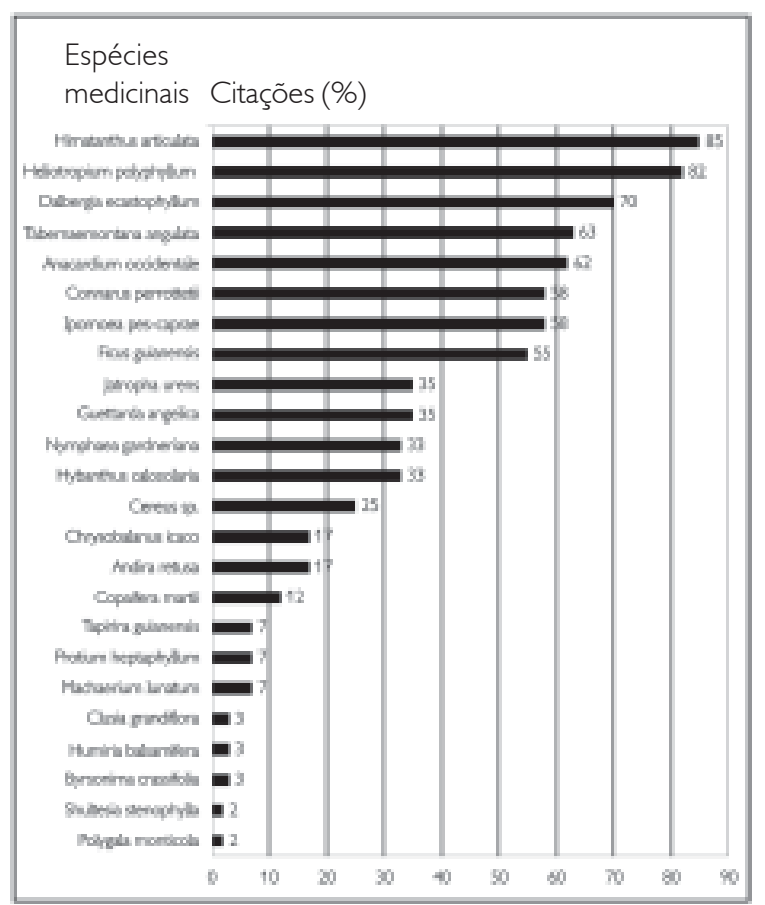

Figura 3. Espécies medicinais levantadas na restinga da Princesa, Algodoal, município de Maracanã, Pará, e a porcentagem dos entrevistados (60) que as citaram. Entrevista estruturada.
(Tabernaemontana angulata), do barbatimão (Connarus perrottettii), da salsa (Ipomoea pes-caprae) e do apuí (Ficus guianensis ), todas citadas por mais de 50\% dos entrevistados. Assim, as duas técnicas complementaram-se, no sentido de verificar as espécies mais importantes para a população de Algodoal e também indicar as menos conhecidas, como o fígado (Schultesia stenophylla) e a jalapinha do mato (Polygala monticola), merecendo especial atenção no que se refere ao resgate do conhecimento.

Com relação à copaíba (Copaifera martii), cujo óleo foi indicado como anti-inflamatório e cicatrizante, não é utilizada e nem mesmo conhecida pela população, que usa o produto (óleo de copaíba) proveniente de outras espécies do gênero, comprado em estabelecimentos comerciais, fato explicado pelos informantes devido ao pequeno diâmetro que as árvores possuem no local estudado.

Deste modo, os resultados evidenciaram que, mesmo fazendo uso de plantas introduzidas de outras localidades, os moradores de Algodoal, de modo geral, ainda conhecem e utilizam bastante as plantas da restinga.

\section{CONCLUSÕES}

A população de pescadores de Algodoal utilizam para fins medicinais 24 espécies, em 19 famílias botânicas, ocorrentes na restinga da Princesa.

Apesar do grande uso, pelos moradores da vila, de plantas exóticas para fins medicinais, foi possível, empregando entrevistas estruturadas, determinar a elevada importância cultural de oito espécies ocorrentes na restinga, todas estas citadas por mais de $50 \%$ dos entrevistados. São elas: Himatanthus articulata, Heliotropium polyphyllum, Dalbergia ecastophyllum, Tabernaemontana angulata, Anacardium occidentale, Connarus perrottettii, Ipomea pes-caprae e Ficus guianensis.

Três espécies da restinga destacaram-se, através do free listing, por estarem entre as dez mais citadas

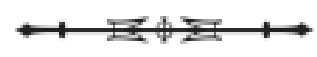


para fins medicinais, nos vários ambientes explorados: Himatanthus articulata, Anacardium occidentale e Dalbergia ecastophyllum.

O emprego, de forma combinada, de duas técnicas usuais em etnobotânica free-listing e entrevista estruturada, apresentou resultados complementares, mostrando-se satisfatório para trabalhos dessa natureza.

Ao demonstrar a importância cultural da flora da restinga da Princesa para a população de Algodoal, esse estudo fornece subsídios para a elaboração do plano de manejo da Área de Proteção Ambiental (APA) na qual se localiza.

\section{AGRADECIMENTOS}

Aos moradores de Algodoal que colaboraram e participaram com informações imprescindíveis para a realização desta pesquisa, a Antônio Elielson Rocha pelo auxílio na formatação do texto e por conceder a figura sobre a localização da área de estudo e a Márlia Regina Coelho Ferreira, da Coordenação de Botânica do Museu Paraense Emílio Goeldi, pelas valiosas sugestões.

\section{REFERÊNCIAS}

ALEXIADES, M. N. 1996. Selected guidelines for ethnobotanical research: a field manual. New York: New York Batanical Garden. 306 p.

ALVES FILHO, A.; ALVES JÚNIOR, J.; MAIA NETO, J. 1999. Pontos de história da Amazônia. 2. ed. Belém: Produção independente. 97 p. v. 1.

AMARAL, D. D. 1997. Contribuição ao estudo das formações abertas de moitas do litoral paraense, Restinga do Crispim, Município de Marapanim - Pará. 170 f. Dissertação (Mestrado) - Universidade Federal do Pará, Museu Paraense Emílio Goeldi, Belém.

AMARAL, D. D. et al. 2001 a. Aspectos taxonômicos de espécies arbustivas e arbóreas ocorrentes em moitas (Restinga do Crispim), Marapanim-PA. Bol. Mus. Para. Emílio Goeldi, sér. Bot., v. 17, n. 1, p. 21-74.

AMARAL, D. D. et al. 2001b. A Vegetação da Iha Canela, Município de Bragança - Pará, Brasil. Bol. Mus. Para. Emílio Goeldi, sér. Bot., v. 17, n. 2, p. 389-402
AMOROZO, M. C. M.; GÉLY, A. 1988. Uso de plantas medicinais por caboclos do Baixo Amazonas. Barcarena, PA, Brasil. Bol. Mus. Para. Emílio Goeldi, sér. Bot., v. 4, n. 1, p. 47-131.

ARAÚJO, D. S. D.; LACERDA, L. D. 1987. A natureza das restingas. Ciência Hoje, v. 6, n. 33, p. 42-48.

BASTOS, M. N. C. 1988. Levantamento florístico em restinga arenosa litorânea na ilha de Maiandeua-Pará. Bol. Mus. Para. Emílio Goeldi, sér. Bot., v. 4, n. 1, p. 159-173.

BASTOS, M. N. C. 1995. A importância das formações vegetais da restinga e do manguezal para as comunidades pesqueiras. Bol. Mus. Para. Emílio Goeldi, sér. Antropol., v. 11, n. 1, p. $41-56$.

BASTOS, M. N. C.; ROSÁRIO, C. S.; LOBATO, L. C. B. 1995. Caracterização fitofisionômica da restinga de Algodoal, Maracanã, Brasil. Bol. Mus. Para. Emílio Goeldi, sér. Bot., v. 11, n. 2, p. 173-197.

BASTOS, M. N. C. 1996. Caracterização das formações vegetais da restinga da Princesa, Ilha de Algodoal-PA. 249 f. Tese (Doutorado) - Universidade Federal do Pará, Museu Paraense Emílio Goeldi, Belém.

BEGOSSI, A. 1998a. Etnobotânica em comunidades caiçaras. In: FONSECA et al. (Org.). Etnobotânica: Bases para conservação. In: CONGRESSO NACIONAL DE BOTÂNICA DO BRASIL, 47., Nova Friburgo. p. 108-119. I workshop brasileiro de etnobotânica e botânica econômica.

BEGOSSI, A. 1998b. Resilience and neotraditional populations: The caiçaras (Atlantic Forest) and caboclos (Amazon). In: BERKES, F; FOLKE, C. (Ed.). Linking Social and Ecological Systems for Resilience and Sustainability. Cambridge: Cambridge University Press. p. 129-157.

BEGOSSI, A.; LEITÃO-FILHO, H. F; RICHERSON, P. J. 1993. Plant uses in a Brazilian fishing community (Búzios Island). Journal of Ethnobiology, v.13, n. 2, p. 233-256.

BRAGA, P. I. S. 1979. Subdivisão fitogeográfica, tipos de vegetação, conservação e inventário florístico da floresta amazônica. Acta Amazonica, v. 9, n. 4, p. 53-80. Suplemento.

COELHO-FERREIRA, M. R. 2000. Identificação e valorização das plantas medicinais de uma comunidade pesqueira do litoral paraense (Amazônia brasileira). 259 f. Tese (Doutorado) - Universidade Federal do Pará, Museu Paraense Emílio Goeldi, Belém.

$\mathrm{COHN}$, J. P. 1988. Culture and conservation. A greater sensitivity to local culture could increase the success of both conservation and development projects. Bio Science, v. 38, n. 7, p. 450-453.

COSTA NETO, S. V. 1999. As formações herbáceas da restinga do Crispim, Marapanim - Pará. 120 f. Dissertação (Mestrado) - Faculdade de Ciências Agrárias do Pará, Belém.

COSTA NETO, S. V.; BASTOS, M. N. C.; LOBATO, L. C. B. 1996. Composição florística e fitofisionomia da restinga do Crispim, município de Marapanim, PA. Bol. Mus. Para. Emílio Goeldi, sér. Bot., v. 12, n. 2, p. 237-249.

COSTA NETO, S. V. et al. 2001. Fitossociologia das Formações Herbáceas da Restinga do Crispim, Marapanim-PA. Bol. Mus. Para. Emílio Goeldi, sér. Bot., v. 17, n. 1, p. 161-186.

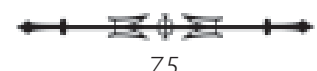


CORRÊA, C. G. et al. 1994. O processo de ocupação humana na Amazônia: Considerações e perspectivas. Bol. Mus. Para. Emílio Goeldi, sér. Antropol., v. 9, n. 1, p. 1-53.

CRONQUIST, A. 1981. An integrated system of classification of flowering plants. New York: Columbia University Press. 1261 p. ENGLER, A.; PRANTL, K. 1894. Leguminosae. Die natürlichen Planzenfamilien. Leipzig: Wilhelm Engelman. p. 70-388. v. 3.

FAJARDO, E. 2002. Sambaquis. As testemunhas atuais da pré-história no Brasil. Ecologia e Desenvolvimento, v. 12, n. 101, p. 12-19. FLEMING-MORAN, M. 1992. The folk view of natural causation and disease in Brazil its relation to traditional curing practices. Bol. Mus. Para. Emílio Goeldi, sér. Antropol., v. 8, n. 1, p. 65-156.

FIGUEIREDO, G. M.; LEITÃO-FILHO, H. F.; BEGOSSI, A. 1993. Ethnobotany of Atlantic Forest coastal communities: Diversity of plant uses in Gamboa (Itacuruçá Island, Brazil). Human Ecology, v. 21, n. 4, p. 419-430.

FIGUEIREDO, G. M.; LEITÃO-FILHO, H. F.; BEGOSSI, A. 1997. Ethnobothany of Atlantic Forest coastal communities II: Diversity of plant uses at Sepetiba bay (SE Brazil). Human Ecology, v. 25, n. 2, p. 353-360.

FONSECA, V. F.; SÁ, C. F. C. 1997. Situación de los estudios de etnobotánica y botánica económica en ecosistemas costeros de Brasil: Nota preliminar. In: RIOS, M.; PEDERSEN, H. B. Memorias del Segundo Simposio Ecuatoriano de Etnobotánica y Botánica Económica. Quito: Ed. Abya-Yala. p. 56-81.

FORD, R. I. 1978. Ethnobotany: Historical diversity and synthesis: In: FORD, R. I.; HODGE, M.; MERRIL, W. L., (Ed.). The Nature and Status of Ethnobotany. Annals of Arnold Arboretum. Michigan: Museum of Anthrpology, University of Michigan. Anthropological Papers, v. 67, p. 33-49.

FUNTEC/SECTAM. 2000. Manejo e Valorização Econômica dos Recursos Naturais da APA de Algodoal/Maiandeua - PA. Relatório Final.

FURTADO, L. G.; SOUZA, R. C.; BERG, M. E. 1978. Notas sobre uso terapêutico de plantas pela população cabocla de Marapanim, Pará. Bol. Mus. Para. Emílio Goeldi, Nova sér. Antropol., v. 70, n. 1, p. 1-31.

FURTADO, L. G. 1981. Pesca artesanal: Um delineamento de sua história no Pará. Bol. Mus. Para. Emílio Goeldi, Nova sér. Antropol., n. 79, p. 1-50.

FURTADO, L. G. 1987. Curralistas e Redeiros de Marudá: Pescadores do Litoral do Pará. Belém: Museu Paraense Emílio Goeldi. 366 p.

GADGIL, M.; BERKES, F.; FOLKE, C. 1993. Indigenous knowledge for biodiversity conservation. Ambio, v. 22, p. 51-156.

GASPAR, M. 2000. Sambaqui: Arqueologia do litoral brasileiro. Rio de Janeiro: Jorge Zahar. 89 p. il. Descobrindo o Brasil.

GOTTLIEB, O. R.; KAPLAN, M. A. C. 1990. Amazônia: Tesouro químico a preservar. Ciência Hoje, v. 11, n. 61, p. 17-20.

HALFFTER, G. 1985. Biosphere Reserves: Conservation of Nature for Man. Parks, v. 10, n. 3, p. 15-18.

KNEIP, L. M. 1987. Sambaquis na pré-história do Brasil. Ciência Hoje, v. 6, p. 33, p. 50-54.
MALDONADO, S. 1995. Dizer marítmo: A antropologia e os pescadores em algumas notas. Bol. Mus. Para. Emílio Goeldi, sér. Antropol., v. 11, n. 2, p. 137-144.

MARTIN, G. J. 1995. Ethnobotany: a methods manual. New. York: Chapman and Hall. 276 p.

MING, L. C. 1995. Levantamento de plantas medicinais na Reserva Extrativista "Chico Mendes". 180 f. Tese (Doutorado) - Universidade Estadual Paulista, Acre.

MING, L. C; DI STASI, L. C. (Ed.). 1996. Coleta de plantas medicinais. In: PLANTAS medicinais: Arte e ciência. São Paulo: UNESP. p. 69-86. Um guia de estudo interdisciplinar.

PARKER, E. P.1989. A Neglected resource in Amazonia. The Amazon caboclo. Resource management in Amazonia: Indigenous and folk strategies. In: POSEY, D.A .; BALÉE, W. (Ed.). Advance in Botany. New York: New York Botany Garden. Bronx. p. 249-259. v. 7.

PRANCE, G T. 1991. What is ethnobotany today? Journal of Ethnopharmacology, v. 32, p. 209-16.

ROCHA, A. E. S. 2000. Poaceae na restinga da Praia da Princesa, Maracanã, Pará, Brasil. 114 f. Dissertação (Mestrado) - Faculdade de Ciência Agrárias do Pará, Belém.

SANTOS, J. U. M. et al. 1997. Levantamento das plantas utilizadas por moradores da Ilha de Maiandeua, Município de Maracanã, Estado do Pará. In: REUNIÃO DOS BOTÂNICOS DA AMAZÔNIA, 2., 1997, Salinópolis, Pará. Programa e Resumos... Belém: Museu Paraense Emílio Goeldi. p. 86.

SANTOS, J. U. M.; ROSÁRIO, C. S. 1988. Levantamento da Vegetação Fixadora das Dunas de Algodoal - PA. Bol. Mus. Para. Emílio Goeldi, sér. Bot., v. 4, n. 1, p. 133-151.

SCHMITZ, P. I. 1989. A história do velho Brasil. Ciência Hoje, v. 9, n. 54, p. $40-48$.

SIMÕES, M. F. 1981. Coletores-Pescadores ceramistas do litoral do Salgado (Pará) - Nota preliminar. Bol. Mus. Para. Emílio Goeldi, Nova sér. Antropol., v. 78, p. 1-26.

URQUIZA, N. G. et al. 1999. Estudo etnobotânico na comunidade de Fortalezinha, Iha de Maiandeua, Município de Maracanã (PA). In: CONGRESSO NACIONAL DE BOTÂNICA. Resumos... Blumenau: [s.n.]. p. 50.

VIANNA, C.; MORGADO, M. A. 2001. Os Encantos e Mistérios da Tha de Algodoal. Pará Onde, v. 6, n. 2, p. 6-13.

XOLOCOTZY, E. H. 1983. El concepto de Etnobotánica. In: BARRERA, A. (Ed.). La Etnobotánica: Tres puntos de vista y una perspectiva. Xalapa: Veracruz. Instituto Nacional de Investigaciones sobre Recursos Bióticos. p. 13-18.
Recebido:19/12/2002 Aprovado: 15/10/2004

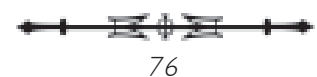


Apêndice 1. Plantas mais utilizadas pela população de Algodoal, município de Maracanã, Pará - Free-listing.

1) Quais as plantas que mais utiliza para fins medicinais?

Apêndice 2. Espécies medicinais da restinga da Princesa mais utilizadas pela população de Algodoal, município de Maracanã, Pará. Entrevista estruturada (acompanhado do herbário-móvel e fotografias).

Nome comum $\quad$ Conhece? Usa? Para quê?

Caju

Tapiririca

Sucuba

Pocoró

Sete-sangrias

Breu-branco

Jamaracaru

Ajiru

Ceboleira

Barbatimão

Salsa

Urtiga-branca

Fígado

Mirim

Copaíba

Andiroba-jaruba

Turiá

Muruci

Apuí

Apé

Jalapinha-do mato

Angélica

Ipecacunha

Verônica 
Apêndice 3. Espécies medicinais da restinga da Princesa, ilha de Algodoal, município de Maracanã, Pará, registradas por familia, nome popular e número de citações.

\begin{tabular}{|c|c|c|c|}
\hline Família & Espécie & Nome Popular & $N^{\circ}$ de citações \\
\hline \multirow[t]{2}{*}{ Anacardiaceae } & Anacardium occidentale L. & Caju & 37 \\
\hline & Tapirira guianensis Aubl. & Tapiririca & 4 \\
\hline \multirow[t]{2}{*}{ Apocynaceae } & Himatanthus articulata (Vahl.) Woodson & Sucuba & 51 \\
\hline & Tabernaemontana angulata Mart. ex M. Arg. & Pocoró & 38 \\
\hline Boraginaceae & Heliotropium polyphyllum var. blanchetti DC. Rich. & Sete-sangrias & 49 \\
\hline Burseraceae & Protium heptaphyllum (Aubl.) March. & Breu-branco & 4 \\
\hline Cactaceae & Cereus sp. & Jamaracaru & 15 \\
\hline Chrysobalanaceae & Chrysobalanus icaco L. & Ajiru & 10 \\
\hline Clusiaceae & Clusia grandiflora Splitgz. & Ceboleira & 2 \\
\hline Connaraceae & Connarus perrottettii (DC) Planch. var. angustifolius Raldeck & Barbatimão & 35 \\
\hline Convolvulaceae & Ipomoea pes-caprae Rottb. & Salsa & 35 \\
\hline Euphorbiaceae & Jatropha urens var. genuina M. Arg. & Urtiga-branca & 21 \\
\hline Gentianaceae & Schultesia stenophylla Mart. & Fígado & 1 \\
\hline Humiriaceae & Humiria balsamifera Aubl. & Mirim & 2 \\
\hline \multirow[t]{4}{*}{ Leguminosae } & Copaifera martii Hayne & Copaíba & 7 \\
\hline & Andira retusa (Lam.) H.B.K. & Andiroba-jaruba & 10 \\
\hline & Machaerium lunatum (L.f.) Ducke & Turiá & 4 \\
\hline & Dalbergia ecastophyllum (L.) Taub. & Verônica & 42 \\
\hline Malpighiaceae & Byrsonima crassifolia (L.) H.B.K. & Muruci & 2 \\
\hline Moraceae & Fícus guianensis Desv. ex Ham. & Apuí & 33 \\
\hline Nymphaeaceae & Nymphaea gardneriana Planch. & Apé & 20 \\
\hline Polygalaceae & Polygala monticola H.B.K. & Jalapinha-do-mato & 1 \\
\hline Rubiaceae & Guettarda angelica Mart. & Angélica & 21 \\
\hline Violaceae & Hybanthus calceolaria (L.) Schulze-Menz & Ipecacunha & 20 \\
\hline
\end{tabular}

Apêndice 4. Espécies medicinais usadas na Vila de Algodoal, município de Maracanã, Pará e número de citações.

\begin{tabular}{llll}
\hline Nome comum & Nome específico & Familia & $N^{\circ}$ de citações \\
\hline Abacate & Persea americana Mill. & 04 \\
Açaí & Euterpe oleracea Mart. & Lauraceae & 01 \\
Alfavaca & Ocimum micranthum Willd. & Arecaceae & 03 \\
Alfavacão & Ocimum cf. selloi Benth & Lamiaceae & 13 \\
Algodão branco & Gossypium herbaceum L. & Lamiaceae & 03 \\
Algodão do Pará & & Malvaceae & 01 \\
Alho & Allium sativum L. & ? & 04 \\
Amor crescido & Portulaca pilosa L. & Liliaceae & 08 \\
Anador & Plectranthus barbatus Benth. & Portulacaceae & 11 \\
Andiroba & Carapa guianensis Aublet. & Lamiaceae & 01 \\
Angélica $*$ & Guettarda angelica Mart. & Meliaceae & 05 \\
Arruda & Ruta graveolens L. & Rubiaceae & 04 \\
Ata & Annona squamosa L. & Rutaceae & 03 \\
Babosa & Aloe vera (L.) Burman & Annonaceae & 01 \\
Barbatimão $*$ & Connarus perrottettii (DC) Planch. var. augustifolius Raldeck & Liliaceae & 08 \\
Batatão & Operculina alata (Harm.) Hub. & Convolvulaceae & 01 \\
Bucha & Luffa cylindrica M. Roem. & Cucurbitaceae & 01 \\
Caabi & Cabi paraensis Ducke & Malpighiaceae & 01 \\
Caju* & Anacardium occidentale L. & Anacardiaceae & 12 \\
\hline
\end{tabular}

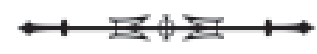


Apêndice 4. Continuação

\begin{tabular}{|c|c|c|c|}
\hline Nome comum & Nome específico & Familia & $N^{\circ}$ de citações \\
\hline Camomila & Matricaria chamomilla L. & Asteraceae & 01 \\
\hline Canarana & Costus spicatus Sw. & Zingiberaceae & 02 \\
\hline Canela & Cinnamomum zeylanicum Nees & Lauraceae & 02 \\
\hline Capim santo & Cymbopogon citratus (DC) Stapf & Poaceae & 07 \\
\hline Carrapato & Ricinus communis $\mathrm{L}$. & Euphorbiaceae & 01 \\
\hline Castanhola & Terminalia catappa L. & Combretaceae & 02 \\
\hline Catinga-de-mulata & Aeollanthus suaveolens Mart. ex Spreng. & Lamiaceae & 04 \\
\hline Chicória & Eryngium foetidum $\mathrm{L}$. & Apiaceae & 01 \\
\hline Cipó d'alho & Adenocalymna alliaceum Miers. & Bignoniaceae & 01 \\
\hline Coco & Cocos nucifera $\mathrm{L}$. & Arecaceae & 02 \\
\hline Copaíba * & Copaifera martii Hayne & Leguminosae & 01 \\
\hline Coramina & Pedilanthus tithymaloides Poit. & Euphorbiaceae & 01 \\
\hline Couve & Brassica oleracea L. & Brassicaceae & 01 \\
\hline Cravo & Tagetes sp. & Asteraceae & 01 \\
\hline Erva-cidreira & Lippia alba (Mill.) N.E.Br. & Verbenaceae & 12 \\
\hline Erva-de-jaboti & Peperomia pellucida (L.) H.B.K. & Piperaceae & 02 \\
\hline Eucalipto & Eucalyptus sp. & Myrtaceae & 08 \\
\hline Figativo & & $?$ & 01 \\
\hline Goiaba & Psidium guajava L. & Myrtaceae & 08 \\
\hline Graviola & Annona muricata L. & Annonaceae & 04 \\
\hline Hortelão & Plectranthus amboinicus (Lour.) Sprengel & Lamiaceae & 09 \\
\hline Hortelãzinho & Mentha pulegium $\mathrm{L}$. & Lamiaceae & 13 \\
\hline Ipecacunha * & Hybanthus calceolaria (L.) Schulze-Menz & Violaceae & 01 \\
\hline Jamaracaru * & Cereus sp. & Cactaceae & 01 \\
\hline Japana & Eupatorium sp. & Asteraceae & 02 \\
\hline Jatobá & Hymenaea courbaril L. & Leguminosae & 04 \\
\hline Jucá & Caesalpinia ferrea Mart. & Leguminosae & 09 \\
\hline Jurubeba & Solanum sp. & Solanaceae & 02 \\
\hline Jutaí & Hymenaea parvifolia L. & Leguminosae & 01 \\
\hline Laranja & Citrus sinensis (L.) Osbeck & Rutaceae & 05 \\
\hline Limão & Citrus sp. & Rutaceae & 05 \\
\hline Limãozinho & Citrus aurantifolia (Christm.) Swingle & Rutaceae & 02 \\
\hline Língua-de-vaca & Elephantopus scaber L. & Asteraceae & 02 \\
\hline Losna & Ambrosia sp & Asteraceae & 01 \\
\hline Mamão & Carica papaya L. & Caricaceae & 03 \\
\hline Manga & Mangifera indica L. & Anacardiaceae & 02 \\
\hline Manjericão & Ocimum minimum $\mathrm{L}$. & Lamiaceae & 19 \\
\hline Manjerona & Origanum majorana L. & Lamiaceae & 01 \\
\hline Mastruz & Chenopodium ambrosioides $\mathrm{L}$. & Chenopodiaceae & 11 \\
\hline Meracilina & & $?$ & 01 \\
\hline Mucura-caá & Petiveria alliacea $\mathrm{L}$. & Phytolaccaceae & 03 \\
\hline Muruci * & Byrsonima crassifolia (L.) H.B.K. & Malpighiaceae & 01 \\
\hline Oriza & Pegostemon heyneanus Benth. & Lamiaceae & 02 \\
\hline Pamarioba & Senna occidentalis Link. & Leguminosae & 02 \\
\hline Parerê & & ? & 01 \\
\hline Pariri & Arrabidaea chica Verl. & Bignoniaceae & 09 \\
\hline Pata-de-vaca & Bauhinia forficata Link. & Leguminosae & 01 \\
\hline Pião-branco & Jatropha curcas L. & Euphorbiaceae & 02 \\
\hline Pirarucu & Bryophyllum calycinum Salisb. & Crassulaceae & 02 \\
\hline
\end{tabular}


Apêndice 4. Continuação

\begin{tabular}{|c|c|c|c|}
\hline Nome comum & Nome específico & Familia & $N^{0}$ de citações \\
\hline Quebra-pedra & Phyllanthus niruri L. & Euphorbiaceae & 06 \\
\hline Quina & Quassia amara L. & Simarubaceae & 01 \\
\hline Sabugueiro & Sambucus nigra L. & Sambucaceae & 01 \\
\hline Sacaca & Vernonia condensata Baker & Asteraceae & 05 \\
\hline Sete sangrias $*$ & Heliotropium polyphyllum var. blanchetti DC. Rich. & Boraginaceae & 04 \\
\hline Sucuba $*$ & Himathantus articulata (Vahl.) Woodson & Apocynaceae & 18 \\
\hline Sucuriju & Mikania lindleyana DC. & Asteraceae & 01 \\
\hline Taperebá & Spondias mombin L. & Anacardiaceae & 02 \\
\hline Urtiga branca * & Jathropha urens var. genuina M. Arg. & Euphorbiaceae & 01 \\
\hline Urucu & Bixa orellana $\mathrm{L}$. & Bixaceae & 02 \\
\hline Verônica * & Dalbergia ecastophyllum (L.) Taub. & Leguminosae & 10 \\
\hline Vindecaá & Alpinia nutans Rosc. & Zingiberaceae & 02 \\
\hline
\end{tabular}

* espécie ocorrente na restinga da Princesa. 